\title{
A REMARK ON THE NON-RATIONALITY PROBLEM FOR GENERIC CUBIC FOURFOLDS
}

\author{
VIK. S. KULIKOV
}

\begin{abstract}
It is proved that the non-rationality of a generic cubic fourfold follows from a conjecture on the non-decomposability in the direct sum of non-trivial polarized Hodge structures of the polarized Hodge structure on transcendental cycles on a projective surface.
\end{abstract}

Before to formulate the main statement of the present note, recall some definitions and statements related to Hodge structures.

Let $\mathcal{H}=\left\{M, H^{p, n-p}, Q\right\}$ be a polarized Hodge structure of weight $n$, that is, $M$ is a free $\mathbb{Z}$-module, $Q: M \times M \rightarrow \mathbb{Z}$ is a non-degenerate bilinear form, the vector space $M \otimes \mathbb{C}=\bigoplus_{p=a}^{b} H^{p, n-p}$ is the direct sum of its complex subspaces $H^{p, n-p}$ for some $a, b \in \mathbb{Z}, a \leq b$, such that $H^{n-p, p}=\overline{H^{p, n-p}}$ and

$$
\begin{aligned}
& Q(u, v)=(-1)^{n} Q(v, u) ; \\
& Q(u, v)=0 \quad \text { for } u \in H^{p, n-p}, v \in H^{p^{\prime}, n-p^{\prime}}, p \neq n-p^{\prime} .
\end{aligned}
$$

A polarized Hodge structure $\mathcal{H}=\left\{M, H^{p, q}, Q\right\}$ is called unimodular if $Q$ is an unimodular bilinear form, that is, if $\left(e_{i}\right)$ is a free basis of $M$, then the determinant of the matrix $A=\left(Q\left(e_{i}, e_{j}\right)\right)$ is equal to \pm 1 . Let $\mathcal{H}_{1}=\left\{M_{1}, H_{1}^{p, q}, Q_{1}\right\}$ and $\mathcal{H}_{2}=\left\{M_{2}, H_{2}^{p, q}, Q_{2}\right\}$ be two polarized Hodge structures of weight $n$. A $\mathbb{Z}$-homomorphism $f: M_{1} \rightarrow M_{2}$ of $\mathbb{Z}$-modules is a morphism of polarized Hodge structures if $Q_{2}(f(u), f(v))=Q_{1}(u, v)$ for all $u, v \in M_{1}$ and $f$ induces a morphism of Hodge structures, that is, $f_{\mathbb{C}}\left(H_{1}^{p, q}\right) \subset H_{2}^{p, q}$ for all $p, q$, where $f_{\mathbb{C}}=f \otimes$ Id. Note that if $f: M_{1} \rightarrow M_{2}$ is a morphism of polarized Hodge structures, then $f$ is an embedding, since $Q_{1}$ and $Q_{2}$ are non-degenerate bilinear forms. We say that $\mathcal{H}_{1}$ is a polarized Hodge substructure of $\mathcal{H}_{2}$ (and will write $\left.\mathcal{H}_{1} \subset \mathcal{H}_{2}\right)$ if the embedding $M_{1} \subset M_{2}$ is a morphism of polarized Hodge structures. A polarized Hodge structure $\mathcal{H}=\left\{M_{1} \oplus M_{2}, H^{p, q}, Q_{1} \oplus Q_{2}\right\}$ is called the direct sum of polarized Hodge structures $\mathcal{H}_{1}$ and $\mathcal{H}_{2}$ if the canonical embeddings $M_{i} \subset M_{1} \oplus M_{2}$ are morphisms of polarized

The work was partially supported by the RFBR (05-01-00455), NWO-RFBR 047.011.2004.026 (RFBR 05-02-89000- $N W O_{a}$ ), INTAS (05-1000008-7805), and by RUM1-2692-MO-05. 
Hodge structures for $i=1,2$. In the case of the direct sum we have $H^{p, q}=H_{1}^{p, q} \oplus H_{2}^{p, q}$ for all $p, q$. We say that a polarized Hodge structure $\mathcal{H}$ is non-decomposable if it is not isomorphic to the direct sum of two non-trivial polarized Hodge structures.

Let $X$ be a smooth projective manifold defined over the field $\mathbb{C}$, $\operatorname{dim}_{\mathbb{C}} X=n$. It is well known that one can associate to $X$ a polarized Hodge structure $\mathcal{H}_{X}=\left\{M_{X}, H^{p, q}, Q\right\}$ of weight $n$, where

$$
M_{X}=H^{n}(X, \mathbb{Z}) /\{\text { torsion }\}
$$

$H^{p, q}=H_{X}^{p, q} \subset H^{n}(X, \mathbb{C})$ are the spaces of harmonic $(p, q)$-forms on $X$, and $Q=Q_{X}$ is the restriction to $M_{X} \subset M_{X} \otimes \mathbb{C} \simeq H^{n}(X, \mathbb{C})$ of the bilinear form

$$
Q(\phi, \psi)=\int_{X} \phi \wedge \psi
$$

It is well known that the lattice $\left(M_{X}, Q_{X}\right)$ is unimodular. Denote by $h^{p, q}=h^{p, q}(X)=\operatorname{dim} H_{X}^{p, q}=\operatorname{dim} H^{q}\left(X, \Omega_{X}^{p}\right)$ the Hodge numbers of $X$, where $\Omega_{X}^{p}$ is the sheaf of holomorphic $p$-forms on $X$.

Lemma 1. Let $f: X \rightarrow V$ be a bi-rational morphism of smooth projective manifolds, $\operatorname{dim}_{\mathbb{C}} X=\operatorname{dim}_{\mathbb{C}} V=n$. Then the polarized Hodge structure $\mathcal{H}_{X}$ is decomposed in the direct sum of the polarized Hodge structure $f^{*}\left(\mathcal{H}_{V}\right) \simeq \mathcal{H}_{V}$ and a polarized Hodge structure $\mathcal{H}_{V}^{\perp}$.

Proof. This statement is well-known in the particular case if $f=\sigma$ : $X \rightarrow V$ is the monoidal transformation with non-singular center $C \subset V$ (1], [3], 6]; see also [2]). By induction, the correctness of lemma follows for a composition $f=\sigma_{m} \circ \cdots \circ \sigma_{1}: X \rightarrow V$ of monoidal transformations $\sigma_{i}: X_{i} \rightarrow X_{i-1}$ with non-singular centers $\widetilde{C}_{i-1} \subset X_{i-1}$, where $X_{0}=V$ and $X_{m}=Y$.

Now, let $f$ be an arbitrary bi-rational morphism. Since $\operatorname{deg} f=1$, then

$$
\int_{V} \phi \wedge \psi=\int_{X} f^{*}(\phi) \wedge f^{*}(\psi)
$$

that is, $f^{*}$ is an embedding of the lattice $M_{V}=H^{4}(V, \mathbb{Z})$ in the lattice $M_{X}=H^{4}(V, \mathbb{Z})$. Therefore $f^{*}: \mathcal{H}_{V} \rightarrow \mathcal{H}_{X}$ is an embedding of the polarized Hodge structure. Since the lattices $f^{*}\left(M_{V}\right)$ and $M_{X}$ are unimodular, there is a sublattice $M_{V}^{\perp}$ of $M_{X}$ such that $M_{X}=f^{*}\left(M_{V}\right) \oplus$ $M_{V}^{\perp}$ is the direct sum of lattices.

Let us show that this decomposition in the direct sum of lattices induces a decomposition $\mathcal{H}_{X}=f^{*}\left(\mathcal{H}_{V}\right) \oplus \mathcal{H}_{V}^{\perp}$ in the direct sum of polarized Hodge structures. For this, since the image of Hodge structure under a morphism of Hodge structures is a Hodge structure, it suffices to show that the natural projection $\mathrm{pr}^{\perp}: M_{X} \otimes \mathbb{C} \rightarrow M_{V}^{\perp} \otimes \mathbb{C}$ is a 
morphism of Hodge structures. To show that $\mathrm{pr}^{\perp}$ is a morphism of Hodge structures, let us consider the bi-rational map $f^{-1}: V \rightarrow X$. By Hironaka Theorem, there is a composition $\sigma=\sigma_{m} \circ \cdots \circ \sigma_{1}: Y \rightarrow V$ of monoidal transformations $\sigma_{i}: Y_{i} \rightarrow Y_{i-1}$ with non-singular centers $\widetilde{C}_{i-1} \subset Y_{i-1}$, where $Y_{0}=V$ and $Y_{m}=Y$, such that $g=f^{-1} \circ \sigma: Y \rightarrow X$ is a bi-rational morphism. We obtain a decomposition

$$
\mathcal{H}_{Y}=\sigma^{*}\left(\mathcal{H}_{V}\right) \oplus \bigoplus_{i=0}^{m-1} \widetilde{\mathcal{H}}_{i}
$$

in the direct sum of polarized Hodge structures, where $\widetilde{\mathcal{H}}_{i}$ is the contribution in $\mathcal{H}_{Y}$ of the monoidal transformation $\sigma_{i+1}$. Note that the natural projection $\widetilde{\mathrm{pr}}_{2}: \mathcal{H}_{Y} \rightarrow \oplus \bigoplus_{i=0}^{m-1} \widetilde{\mathcal{H}}_{i}$ is a morphism of polarized Hodge structures.

It follows from the commutative diagram

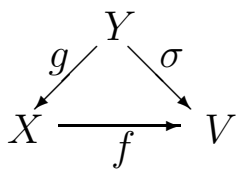

that $g^{*}: \mathcal{H}_{X} \rightarrow \mathcal{H}_{Y}$ is an embedding of polarized Hodge structures, such that $g^{*}\left(f^{*}\left(\mathcal{H}_{V}\right)\right)=\sigma^{*}\left(\mathcal{H}_{V}\right)$ and $g^{*}\left(M_{V}^{\perp}\right) \subset\left(\sigma^{*}\left(M_{V}\right)\right)^{\perp}$. Therefore we can identify $\mathcal{H}_{X}$ with its image $g^{*}\left(\mathcal{H}_{X}\right), f^{*}\left(\mathcal{H}_{V}\right)$ with $\sigma^{*}\left(\mathcal{H}_{V}\right)$, and $M_{V}^{\perp}$ with $g^{*}\left(M_{V}^{\perp}\right)$. Under this identification, the projection $\mathrm{pr}^{\perp}$ is identified with the restriction of $\widetilde{\mathrm{pr}}_{2}$ to $g^{*}\left(\mathcal{H}_{X}\right)$. Therefore $\mathrm{pr}^{\perp}$ is a morphism of Hodge structures as the composition of two morphisms of Hodge structures: namely, of the embedding $g^{*}$ and the projection $\widetilde{\mathrm{pr}}_{2}$.

Let $\operatorname{dim}_{\mathbb{C}} X=2 k$. Believing in Hodge Conjecture, the elements of $A_{X}=M_{X} \cap H^{k, k}$ will be called algebraic and the module $T_{X}=$ $\left\{\gamma \in M_{X} \mid Q(\gamma, \alpha)=0\right.$ for all $\left.\alpha \in A_{X}\right\}$ will be called the module of transcendental $n$-cycles on $X, n=2 k$. It is easy to see that the polarized Hodge structure $\mathcal{H}_{X}$ induces a polarized Hodge structure $\mathcal{T}_{X}=\left\{T_{X}, H_{T}^{p, q}, Q_{T}\right\}$, where $H_{T}^{p, q}=(T \otimes \mathbb{C}) \cap H_{X}^{p, q}$ and $Q_{T}$ is the restriction of $Q$ to $T_{X}$.

If $S$ is a smooth projective surface, then the form $Q=Q_{S}$ is symmetric unimodular and, by Index Theorem, its signature is equal to $\left(2 h^{2,0}+1, h^{1,1}-1\right)$. Note that the polarized Hodge structure $\mathcal{T}_{S}$ on the transcendental cycles on a smooth projective surface $S$ is a bi-rational invariant of the surface $S$. 
Non-decomposability Conjecture. The polarized Hodge structure $\mathcal{T}_{S}$ on the transcendental cycles on a smooth projective surface $S$ is non-decomposable.

Let $V \subset \mathbb{P}^{5}$ be a smooth cubic fourfold. It is known (see [8] and 9) that the moduli space of cubic fourfolds contains several families of rational cubics. Nevertheless, the following conjecture is well known.

Non-rationality Conjecture. A generic cubic fourfold is non-rational.

The aim of the present note is to show that Non-rationality Conjecture follows from Non-decomposability Conjecture. To formulate the precise statement, we fix one of smooth cubic fourfolds $V_{0}$. For each cubic fourfold $V$ we can identify the lattice $\left(M_{V}, Q_{V}\right)$ with the lattice $\left(M_{0}, Q\right)$, where $M_{0}=H^{4}\left(V_{0}, \mathbb{Z}\right)$ and $Q=Q_{V_{0}}$. Let $\lambda=L^{2} \in$ $M_{0}$, where $L \in H^{2}\left(V_{0}, \mathbb{Z}\right)$ is the class of the hyperplane section of $V_{0} \subset \mathbb{P}^{5}$. It is well known (see, for example, 7]) that each smooth cubic fourfold $V$ has the following Hodge numbers: $h^{4,0}=h^{0,4}=0$, $h^{3,1}=h^{1,3}=1$, and $h^{2,2}=21$. Consider the polarized Hodge structures $\mathcal{H}_{V}=\left\{M_{V}, H^{p, q}, Q_{V}\right\}$ on the fourth cohomology groups of the smooth cubic fourfolds $V$. Since $h^{4,0}=0$ and $h^{3,1}=1$, the polarized Hodge structure $\mathcal{H}_{V}=\left\{M_{0}, H_{V}^{p, q}, Q\right\}$ is defined by a non-zero element $\omega \in H_{V}^{3,1} \subset M_{0} \otimes \mathbb{C}$. It follows from Hodge - Riemann bilinear relations that $Q(\lambda, \omega)=Q(\omega, \omega)=0$ and $Q(\omega, \bar{\omega})<0$. Therefore the classifying space $D$ of polarized Hodge structures of the smooth cubic fourfolds coincides with

$$
D=\left\{\omega \in \mathbb{P}^{22} \mid Q(\omega, \lambda)=Q(\omega, \omega)=0, Q(\omega, \bar{\omega})<0\right\} .
$$

The point $\omega(V) \in D$, corresponding to the polarized Hodge structure $\mathcal{H}_{V}=\left\{M_{0}, H^{p, q}(V), Q\right\}$ of a smooth cubic fourfold $V$, is called the periods of $V$. By [4] and by Global Torelli Theorem proved in [10], the set $D_{0}$ of periods of the smooth cubic fourfolds is an open subset of $D$.

Let $P=\left\{\mu \in M_{0} \mid Q(\lambda, \mu)=0\right\}$ be the submodule of $M_{0}$ consisting of the primitive elements. For each endomorphism $A \in \operatorname{End}\left(M_{0}\right)$ such that $A(P) \subset P$ and the restriction $A_{\mid P}$ of $A$ to $P$ is not proportional to the identity automorphism of $P$, denote by

$$
E_{A}=\left\{\omega \in D \subset \mathbb{P}^{22} \mid \omega \text { is an eigenvector of } A\right\} .
$$

Obviously, $E_{A}$ is the intersection of $D$ and a finite number of linear subspaces of $\mathbb{P}^{22}$ of codimension at least 2 . Therefore $E_{A}$ is a proper closed analytic subvariety of $D$. Put $E=\cup E_{A}$, then $E$ is the union 
of countably many proper closed analytic subvarieties of $D$. Therefore $D \backslash E$ is everywhere dense in $D$.

For each $\mu \in M_{0}, \mu$ is not proportional to $\lambda$, we put

$$
B_{\mu}=\left\{\omega \in D \subset \mathbb{P}^{22} \mid Q(\mu, \omega)=0\right\}
$$

and $B=\cup B_{\mu}$. Similarly to above, $B$ is the union of countably many proper closed analytic subvarieties of $D$. Therefore $D_{0} \backslash(E \cup B)$ is also everywhere dense in $D_{0}$.

Proposition 1. If Non-decomposability Conjecture is true, then a smooth cubic fourfold $V$ is non-rational if its periods $\omega(V) \in D_{0} \backslash(E \cup B)$.

Proof. Assume that a smooth cubic fourfold $V$ is rational and its periods $\omega(V) \in D_{0} \backslash(E \cup B)$.

Note that the sublattice of transcendental elements $T_{V}$ of the lattice $M_{V}$ coincides with the sublattice of primitive elements $P$ if the periods $\omega(V) \in D_{0} \backslash B$.

Since $V$ is rational, then there is a bi-rational map $r: \mathbb{P}^{4} \rightarrow V$. By Hironaka Theorem, there is a composition $\tau=\tau_{n} \circ \cdots \circ \tau_{1}: X \rightarrow \mathbb{P}^{4}$ of monoidal transformations $\tau_{i}: X_{i} \rightarrow X_{i-1}$ with non-singular centers $C_{i-1} \subset X_{i-1}$, where $X_{0}=\mathbb{P}^{4}$ and $X_{n}=X$, such that $f=r \circ \tau: X \rightarrow V$ is a bi-rational morphism.

Each $\tau_{i}$ induces an inclusion of unimodular polarized Hodge structures $\tau_{i}^{*}: \mathcal{H}_{X_{i-1}} \hookrightarrow \mathcal{H}_{X_{i}}$ such that $\mathcal{H}_{X_{i}} \simeq \tau_{i}^{*}\left(\mathcal{H}_{X_{i-1}}\right) \oplus\left(\tau_{i}^{*}\left(\mathcal{H}_{X_{i-1}}\right)^{\perp}\right.$. In particular,

$$
\left.H^{4}\left(X_{i}, \mathbb{Z}\right)\right)=\left(\tau _ { i } ^ { * } ( H ^ { 4 } ( X _ { i - 1 } , \mathbb { Z } ) ) \oplus \left(\tau_{i}^{*}\left(H^{4}\left(X_{i-1}, \mathbb{Z}\right)\right)^{\perp} .\right.\right.
$$

Moreover, if $\operatorname{dim} C_{i-1} \leq 1$, then $\left(\tau_{i}^{*}\left(H^{4}\left(X_{i-1}, \mathbb{Z}\right)\right)^{\perp} \subset H_{X_{i}}^{2,2}\right.$, and if $C_{i-1}$ is a smooth surface, then $\left(\tau_{i}^{*}\left(\mathcal{H}_{X_{i-1}}\right)^{\perp} \simeq-\mathcal{H}_{C_{i-1}}(-1)\right.$, where

$$
-\mathcal{H}_{C_{i-1}}(-1)=\left(M_{C_{i-1}}, H_{i-1}^{p, q},-Q_{C_{i-1}}\right)
$$

and $H_{i-1}^{p, q}=H_{C_{i-1}}^{p-1, q-1}$. Therefore $H_{X_{i}}^{3,1}=\tau_{i}^{*}\left(H_{X_{i-1}}^{3,1}\right) \oplus H_{i-1}^{3,1}, H_{X_{i}}^{4,0}=0$, and $Q_{X_{i}}(u, v)=0$ for $u \in \tau_{i}^{*}\left(H_{X_{i-1}}^{3,1} \oplus H_{X_{i-1}}^{1,3}\right), v \in H_{i-1}^{3,1} \oplus H_{i-1}^{1,3}$.

As a consequence, we obtain a decomposition of the unimodular polarized Hodge structure

$$
\mathcal{H}_{X}=\tau^{*}\left(\mathcal{H}_{\mathbb{P}^{4}}\right) \oplus \bigoplus_{i=0}^{n-1} \mathcal{H}_{i}
$$

in the direct sum of polarized Hodge structures, where $\mathcal{H}_{i}$ is the contribution in $\mathcal{H}_{X}$ of the $(i+1)$ th monoidal transformation. By induction, we have $\mathcal{H}_{i} \simeq\left(\tau_{i}^{*}\left(\mathcal{H}_{X_{i-1}}\right)^{\perp}\right.$ and, consequently,

$$
\mathcal{T}_{X} \simeq-\oplus \mathcal{T}_{C_{i}}(-1)
$$


where the sum is taken over all surfaces $C_{i}$ with $p_{g} \geq 1$, since $H^{4}\left(\mathbb{P}^{4}, \mathbb{C}\right)=$ $H_{\mathbb{P}^{4}}^{2,2}, h^{2,2}\left(\mathbb{P}^{4}\right)=1$, and $H^{2}\left(C_{i}, \mathbb{C}\right)=H_{C_{i}}^{1,1}$ if the geometric genus $p_{g}$ of the surface $C_{i}$ is equal to zero.

The morphism $f$ induces a morphism of Hodge structures $f^{*}: \mathcal{H}_{V} \rightarrow$ $\mathcal{H}_{X}$. By Lemma 1 , the Hodge structure $\mathcal{H}_{X}$ is decomposed in the direct sum $f^{*}\left(\mathcal{H}_{V}\right) \oplus \mathcal{H}_{V}^{\perp}$ of the polarized Hodge structures.

Lemma 2. Let $V$ be a smooth cubic fourfold from Proposition 1 and let $\tau=\tau_{n} \circ \cdots \circ \tau_{1}: X \rightarrow \mathbb{P}^{4}$ and $f=r \circ \tau: X \rightarrow V$ be morphisms described above. Then there is $i_{0}$ such that the polarized Hodge structure $\mathcal{T}_{C_{i_{0}}}$ on the transcendental cycles of the surface $C_{i_{0}}$ is decomposed in the direct sum of polarized Hodge structures $\mathcal{T}_{C_{i_{0}}}^{\prime} \simeq-f^{*}\left(\mathcal{T}_{V}\right)(1)$ and $\mathcal{T}_{C_{i_{0}}}^{\prime \prime}$.

Proof. We have two decompositions

$$
\mathcal{H}_{X}=f^{*}\left(\mathcal{H}_{V}\right) \oplus \mathcal{H}_{V}^{\perp}=\tau^{*}\left(\mathcal{H}_{\mathbb{P}^{4}}\right) \oplus \bigoplus_{i=0}^{n-1} \mathcal{H}_{i}
$$

of the polarized Hodge structure $\mathcal{H}_{X}$. Consider a non-zero element $\omega \in f^{*}\left(H_{V}^{3,1}\right)$. It can be represented in the form

$$
\omega=\sum_{i=0}^{n-1} \omega_{i}
$$

where $\omega_{i} \in H_{i}^{3,1}$. Let $i_{0}$ be an index such that $\omega_{i_{0}} \neq 0$. Put $\omega_{i_{0}}^{\perp}=$ $\sum_{i \neq i_{0}} \omega_{i}$ and let $\operatorname{pr}_{i_{0}}: \mathcal{H}_{X} \rightarrow \mathcal{H}_{i_{0}}, \operatorname{pr}_{i_{0}}^{\perp}: \mathcal{H}_{X} \rightarrow \oplus_{i \neq i_{0}} \mathcal{H}_{i}$, pr: $\mathcal{H}_{X} \rightarrow$ $f^{*}\left(\mathcal{H}_{V}\right), \operatorname{pr}^{\perp}: \mathcal{H}_{X} \rightarrow \mathcal{H}_{V}^{\perp}$ be the natural projections. Note that all these projections are defined over $\mathbb{Z}$. We have $\operatorname{pr}\left(\operatorname{pr}_{i_{0}}(\omega)\right)=a \omega$ for some $a \in \mathbb{C}$, since $\operatorname{dim} f^{*}\left(H_{V}^{3,1}\right)=1$ and $\mathrm{pr}, \operatorname{pr}_{i_{0}}$ are morphisms of Hodge structures.

Let us show that $a=1$. Indeed, the restriction of $\mathrm{pr} \circ \mathrm{pr}_{i_{0}}$ to $f^{*}\left(M_{V}\right) \simeq M_{V}$ induces an endomorphism of $M_{V}$ such that

$$
\operatorname{pr} \circ \operatorname{pr}_{i_{0}}\left(f^{*}(P)\right) \subset f^{*}(P),
$$

since $\mathrm{pr} \circ \mathrm{pr}_{i_{0}}$ is a morphism of Hodge structures, $M_{V} \cap H_{V}^{2,2}=\mathbb{Z} \lambda$ and $P=T_{V}$ by assumption. Therefore $\left(\operatorname{pr} \circ \operatorname{pr}_{i_{0}}\right)_{\mid f^{*}(P)}=a \cdot \mathrm{Id}$, since $\operatorname{pr}\left(\operatorname{pr}_{i_{0}}(\omega)\right)=a \omega$ (that is, $\omega$ is an eigenvector of $\left(\operatorname{pr} \circ \operatorname{pr}_{i_{0}}\right)_{\mid f^{*}\left(M_{V}\right)}$ and $a$ is its eigenvalue) and, by assumption, $\omega(V) \in D_{0} \backslash(E \cup B)$. Therefore $a \in \mathbb{Z}$, since $\left(\operatorname{pr} \circ \operatorname{pr}_{i_{0}}\right)_{\mid f^{*}(P)}$ is an endomorphism of $\mathbb{Z}$-module $f^{*}(P) \simeq$ $P$. Let $\omega^{\perp}=\operatorname{pr}^{\perp}\left(\omega_{i_{0}}\right) \in H_{X}^{3,1}$. Then we have

$$
\begin{aligned}
& \omega_{i_{0}}=a \omega+\omega^{\perp}, \\
& \omega_{i_{0}}^{\perp}=(1-a) \omega-\omega^{\perp} .
\end{aligned}
$$


By Hodge - Riemann bilinear relations, $Q_{X}(\gamma, \bar{\gamma}) \leq 0$ for $\gamma \in H_{X}^{3,1}$ and $Q_{X}(\gamma, \bar{\gamma})=0$ if and only if $\gamma=0$. Therefore, without loss of generality, we can assume that $Q_{X}(\omega, \bar{\omega})=-1$ and $Q_{X}\left(\omega^{\perp}, \bar{\omega}^{\perp}\right)=b \leq$ 0 . We have $Q_{X}\left(\omega_{i_{0}}, \bar{\omega}_{i_{0}}\right)=-a^{2}+b$ and, consequently, $a$ and $b$ can not simultaneously be equal to zero, since $\omega_{i_{0}} \neq 0$. Besides, we have

$Q_{X}\left(\omega, \omega^{\perp}\right)=Q_{X}\left(\omega, \bar{\omega}^{\perp}\right)=Q_{X}\left(\bar{\omega}, \omega^{\perp}\right)=Q_{X}\left(\bar{\omega}, \bar{\omega}^{\perp}\right)=Q_{X}\left(\omega_{i_{0}}, \bar{\omega}_{i_{0}}^{\perp}\right)=0$.

Therefore

$$
Q_{X}\left(\omega_{i_{0}}, \bar{\omega}_{i_{0}}^{\perp}\right)=Q_{X}\left(a \omega+\omega^{\perp},(1-a) \bar{\omega}-\bar{\omega}^{\perp}\right)=a(1-a)(-1)-b=0,
$$

that is, $a^{2}-a-b=0$, and hence

$$
a=\frac{1 \pm \sqrt{1+4 b}}{2} .
$$

But, $a \in \mathbb{Z}$ and $b \leq 0$, therefore $b=0, a=1$ and hence $\omega^{\perp}=0$, since $Q_{X}\left(\omega^{\perp}, \bar{\omega}^{\perp}\right)=b=0$. Therefore, $\omega \in H_{i_{0}}^{3,1}$. Besides, we showed that $\left(\operatorname{pr} \circ \operatorname{pr}_{i_{0}}\right)_{\mid f^{*}(P)}=\mathrm{Id}$.

Let us show that $f^{*}\left(T_{V}\right) \subset T_{i_{0}}$. As above, we have two decompositions

$$
\mathcal{T}_{X}=f^{*}\left(\mathcal{T}_{V}\right) \oplus f^{*}\left(\mathcal{T}_{V}\right)^{\perp}=\bigoplus_{i=0}^{n-1} \mathcal{T}_{i}
$$

of the polarized Hodge structure $\mathcal{T}_{X}$. Each $\gamma \in f^{*}\left(T_{V}\right)$ can be written in the form $\gamma=\gamma_{i_{0}}+\gamma_{i_{0}}^{\perp}$, where $\gamma_{i_{0}}=\operatorname{pr}_{i_{0}}(\gamma) \in T_{i_{0}}$ and $\gamma_{i_{0}}^{\perp}=\operatorname{pr}_{i_{0}}^{\perp}(\gamma) \in$ $\oplus_{i \neq i_{0}} T_{i}$. Then, since $\left(\operatorname{pr} \circ \operatorname{pr}_{i_{0}}\right)_{\mid f^{*}(P)}=\operatorname{Id}$ and $P=T_{V}$, we have

$$
\begin{aligned}
& \gamma_{i_{0}}=\gamma+\gamma^{\perp}, \\
& \gamma_{i_{0}}^{\perp}=-\gamma^{\perp},
\end{aligned}
$$

where $\gamma^{\perp}=\operatorname{pr}^{\perp}\left(\gamma_{i_{0}}\right) \in f^{*}\left(T_{V}\right)^{\perp}$. Denote by $p_{(3,1)}: M_{X} \otimes C \rightarrow H_{X}^{3,1}$ the natural projection. Then, by definition of transcendental cycles, $p_{(3,1)}(\gamma) \neq 0$ for each non-zero element $\gamma \in T_{X}$, since the Hodge number $h^{4,0}(X)=0$. In particular, $p_{(3,1)}(\gamma)=a_{\gamma} \omega$ for some $a_{\gamma} \in \mathbb{C}, a_{\gamma} \neq 0$, since $f^{*}\left(H_{V}^{3,1}\right)=\mathbb{C} \omega$. Therefore $p_{(3,1)}\left(\gamma_{i_{0}}\right)=a_{\gamma} \omega$, since $\omega \in f^{*}\left(H_{V}^{3,1}\right) \subset$ $H_{i_{0}}^{3,1}$, and hence $p_{(3,1)}\left(\gamma_{i_{0}}^{\perp}\right)=0$. From this, we have $\gamma_{i_{0}}^{\perp}=0$, that is, $f^{*}\left(T_{V}\right) \subset T_{i_{0}}$.

As a consequence, we obtain that

$$
\mathcal{T}_{i_{0}}=f^{*}\left(\mathcal{T}_{V}\right) \oplus \mathcal{T}_{i_{0}}^{\prime \prime}
$$

is the direct sum of polarized Hodge structures, where $T_{i_{0}}^{\prime \prime}$ is a polarized Hodge substructure of $f^{*}\left(\mathcal{T}_{V}\right)^{\perp}$. To complete the proof of Lemma 2, recall that $\mathcal{T}_{i_{0}} \simeq-\mathcal{T}_{C_{i_{0}}}(-1)$, where $\mathcal{T}_{C_{i_{0}}}$ is the polarized Hodge structure on the transcendental 2-cycles on the smooth projective surface $C_{i_{0}}$. 
Lemma 3. There does not exist a smooth projective surface $S$ such that $\mathcal{T}_{V} \simeq-\mathcal{T}_{S}(-1)$, where $V$ is a smooth cubic fourfold whose periods $\omega(V) \in D_{0} \backslash B$.

Proof. If $\mathcal{T}_{V} \simeq-\mathcal{T}_{S}(-1)$ for some surface $S$, then its geometric genus $p_{g}=h^{2,0}(S)=h^{3,1}(V)=1$ and $\operatorname{rk} T_{S}=\operatorname{rk} T_{V}=22$. Since $p_{g}=1$, the surface $S$ is not a ruled surface. Note that the lattice $T_{S}$ is a bi-rational invariant. Therefore we can assume that $S$ is a minimal model and its second Betti number $b_{2}(S)=h^{1,1}(S)+2 h^{2,0}(S) \geq 23$, since $\operatorname{rk} T_{S}=\operatorname{rk} T_{V}=22$ and $S$ should have also algebraic 2-cycles. Therefore $h^{1,1}(S)$ should be greater than 20, since $h^{2,0}(S)=1$.

On the other hand, it follows from the classification of algebraic surfaces that $K_{S}^{2} \geq 0$, where $K_{S}$ is the canonical class of $S$. Denote by $\chi(S)=1-h^{1,0}(S)+h^{2,0}(S)$ the algebraic Euler characteristic of the surface $S$ and by $e(S)=2+h_{S}^{1,1}+2 h^{2,0}(S)-4 h^{1,0}(S)$ its topological Euler characteristic. Note that $h^{1,0}(S)=\operatorname{dim} H^{0}\left(S, \Omega_{S}^{1}\right) \geq 0$. By Noether formula, we have $\chi(S)=\frac{K_{S}^{2}+e(S)}{12}$ and hence

$$
h^{1,1}(S)=10+10 h^{2,0}(S)-8 h^{1,0}(S)-K_{S}^{2}=20-8 h^{1,0}(S)-K_{S}^{2},
$$

since $h^{2,0}(S)=1$. Therefore $h^{1,1}(S) \leq 20$.

It follows from Lemma 3 that in decomposition (11), the summand $\mathcal{T}_{i_{0}}^{\prime \prime}$ is non-trivial which contradicts Non-decomposability Conjecture.

\section{REFERENCES}

[1] Aeppli A., Modifikation von reellen und komplexen Mannigfaltigkeiten. Comment. Math. Helv., 31 (1957), 219-301.

[2] Clemens C.H. and Griffiths P., The intermediate Jacobian of cubic threefold. Ann. Math., 95 (1972), 281-356.

[3] Deligne P., Milne J.S., Ogus A., and Shih K., Hodge cycles, motives, and Shimura varieties. Lect. Notes Math. 900, (1982), Springer-Verlag, Berlin, Heidelberg, New York.

[4] Griffiths P., On periods of certain rational integrals. I, II. Ann. Math., 90 (1969), 460-495; 496-541.

[5] Griffiths P. and Harris J., Principles of algebraic geometry. A WileyInterscience Publication, John Wiley \& Sons, New York Chichester Brisbane Toronto, 1978.

[6] Katz N., Étude cohomologique des pinceaux de Lefschetz. SGA 7, Exposé XVIII, Lect. Notes Math. 340, (1973), 254-326, SpringerVerlag, Berlin, Heidelberg, New York.

[7] Kulikov Vik.S. and Kurchanov P.F., Complex algebraic varieties: periods of integrals and Hodge structures. Encyclopedia of Math. Sciences, Vol. 36, (1998), 1-217, Springer-Verlag, Berlin, Heidelberg, New York.

[8] Tregub, S. L., Three constructions of rationality of a cubic fourfold. Moscow Univ. Math. Bull. 39 (1984), no. 3, 8-16. 
[9] Tregub, S. L., Two remarks on four-dimensional cubics. (Russian) Uspekhi Mat. Nauk 48 (1993), no. 2(290), 201-202; translation in Russian Math. Surveys 48 (1993), no. 2, 206-208.

[10] Voisin, C. Théorème de Torelli pour les cubiques de $\mathbb{P}^{5}$. Invent. Math., 86, (1986), 577-601.

Steklov Mathematical Institute, Gubkina str., 8, 119991 Moscow, RUSSIA

E-mail address: kulikov@mi.ras.ru 